\title{
Strong evidence for the effectiveness of resin based sealants
}

\author{
Abstracted from \\ Ahovuo-Saloranta A, Forss H, Walsh T et al. \\ Sealants for preventing dental decay in the permanent teeth. \\ Cochrane Database Syst Rev. 2013; Issue 3. Art. No. CD001830. DOI: 10.1002/14651858.CD001830.pub4. \\ Address for correspondence: Luisa Fernandez Mauleffinch, Review Group Co-ordinator, \\ Cochrane Oral Health Group, MANDEC, School of Dentistry, University of Manchester, \\ Higher Cambridge Street, Manchester, M15 6FH, UK. E-mail: luisa.fernandez@manchester.ac.uk
}

\section{Question: What are the effects of different types of fissure sealants in preventing caries in permanent teeth in children and adolescents?}

Data sources Cochrane Oral Health Group's Trials Register, CENTRAL, Medline via OVID, EMBASE via OVID; SCISEARCH, CAplus, INSPEC, NTIS and PASCAL via STN Easy and DARE, NHS EED, HTA (all to September/ November 2012) and ClinicalTrials.gov (to July 2012). There were no restrictions on language or date of publication. Study selection Randomised and quasi-randomised controlled trials of at least 12 months duration comparing no sealant with sealant, or different types of sealants, for preventing caries of occlusal or approximal surfaces of premolar or molar teeth in children and adolescents under 20 years of age.

Data extraction and synthesis Screening of search results, data extraction and assessment of trial quality (using GRADE methods) were by two reviewers independently.

Results There were 34 trials of children aged five to 16 years, with 12 trials (2575 participants) comparing sealants with no sealant, 21 trials (3202 participants) comparing one sealant with another and one trial (752 participants) comparing two types of sealant with no sealant.

Resin sealants compared with no sealants prevented caries in the first permanent molars of children five to 10 years old (six trials at low risk of bias with two years follow up), (odds ratio (OR) $0.12,95 \%$ confidence interval $(\mathrm{Cl}) 0.07$ to 0.19$)$. At 48 to 54 months follow-up, the caries preventive effect was maintained (OR $0.21,95 \% \mathrm{Cl} 0.16$ to 0.28 ) although there were only four trials (two were at low and two at high risk of bias).

No conclusions could be drawn as to whether glass ionomer sealants compared with no sealants prevented caries at 2 year follow-up. The mean difference in DFS was $-0.18,95 \% \mathrm{Cl}-0.39$ to 0.03 .

The relative effectiveness of one type of sealant compared to a different type of sealant was inconclusive as there was great variation in comparisons, outcomes, times of outcomes and background fluoride levels in the 21 studies. There was insufficient evidence for the relative superiority of glass ionomer and resin sealants (very low

This paper is based on a Cochrane Review published in the Cochrane Library 2013, issue 3 (see www.thecochranelibrary.com for information). Cochrane Reviews are regularly updated as new evidence emerges and in response to feedback, and the Cochrane Library should be consulted for the most recent version of the review. event rate in many of the 15 trials). There were inconsistent results for resin-modified glass ionomer sealants compared with resin sealants. No difference in caries increments were found in the two small trials of polyacid-modified resin sealants compared with resin sealants. Conclusions Sealants compared with no sealants, on the occlusal surfaces of permanent molars in children and adolescents, are effective at reducing caries up to 48 months. There is less evidence for longer term follow-up and little for the relative effectiveness of sealing in less high caries risk children. No conclusions could be drawn on the relative effectiveness of different types of sealants.

\section{Commentary}

This is the most recent update of this very significant Cochrane review on sealants for dental caries prevention in the permanent teeth of young people. This review was first reported in $1999^{1}$ and most recently updated in $2008 .^{2}$ As with the previous reviews this one assessed caries prevention, the effectiveness of differing materials, and sealant retention. In addition it examined the use of sealants on approximal surfaces for caries prevention but not lesion infiltration or caries arrest. Although no trials on approximal sealing for caries prevention were actually included in the results. The rigour of the reporting has improved but this does make the review less accessible.

In total 34 trials were included in the review. As with the previous reviews there was strong evidence for the effectiveness of resin sealants (12 trials). After 48-54 months, the retention of resin sealants was in general $70 \%$. Based on the results of six studies with a caries incidence in the molar teeth of $40 \%$, the application of resin sealants would reduce this to $6 \%$ developing caries. It should be remembered that the vast majority of trials examine the effect of a one time application of sealant but in clinical practice sealants should be maintained and repaired if defective; if maintained the effectiveness would be even higher. .

Glass ionomer sealants had much poorer retention, but perhaps due to the low caries incidence in the populations studied, it was not possible for the review to make firm recommendations as to the effectiveness of glass ionomer in general and compared to resin sealants, in particular. However, given the number of studies supporting resin, this must remain the first choice material, with glass ionomer being reserved for use as an interim sealant when co-operation levels or tooth eruption status makes achieving adequate isolation difficult. No adverse effects were reported in the two trials that reported this issue. 
In conclusion this review confirms the effectiveness of resin sealants. There is a need for more trials looking at the effectiveness of differing materials, especially glass ionomers, perhaps focussing on their use in less than ideal situations, because we already know resins work when isolation is not an issue.

Chris Deery

Academic Unit of Oral Health and Development, School of Clinical Dentistry, University of Sheffield, Sheffield, UK
1. Ahovuo-Saloranta A, Hiiri A, Nordblad A, Mäkëla M, Murtomaa H. Pit and fissure sealants for preventing dental decay in the permanent teeth of children and adolescents. Cochrane Database Syst Rev 1999, Issue 4. [DOI: 10.1002/14651858. CD001830]

2. Ahovuo-Saloranta A, Hiiri A, Nordblad A, Mäkelä M, Worthington HV. Pit and fissure sealants for preventing dental decay in the permanent teeth of children and adolescents. Cochrane Database Syst Rev 2008, Issue 4. [DOI: 10.1002/14651858. CD001830.pub3]

3. Deery C. Caries detection and diagnosis, sealants and management of the possibly carious fissure. Brit Dent / 2013; 214: 551-557.

Evidence-Based Dentistry (2013) 14, 69-70. doi:10.1038/sj.ebd.6400945 